\title{
The vector optimization and modeling of a technical systems
}

\author{
Leonid B. Matusov ${ }^{1}$ * \\ ${ }^{1}$ Russian Academy of Science, Institute of Machines Science named after A.A. Blagonravov, \\ Moscow, Russia
}

\begin{abstract}
The construction a feasible solution set with a given accuracy is a main problem in multicriteria optimization and modeling. In order to construct the feasible solution set, a method called the Parameter Space Investigation has been created and successfully integrated into various fields of industry, science, and technology. Multicriteria modeling (identification) is a new direction that is of great value in applications. In the most common usage, the term "identification" means construction of the mathematical model of a system and determination of the parameters (design variables) of the model. The construction a feasible solution set with a given accuracy is a common way for solving multicriteria optimization and modeling problems. The issues of the estimation of the Parameter Space Investigation method convergence rate, the approximation of the feasible solution set are described. Besides these, the multicriteria identification problems of mechanical systems are discussed too.
\end{abstract}

\section{Introduction}

Let us consider a technical system whose operation is described by a system of equations or whose performance criteria may be directly calculated. We assume that the system depends on $r$ design variables $\alpha_{1}, \ldots, \alpha_{r}$ representing a point $\alpha=\left(\alpha_{1}, \ldots, \alpha_{r}\right)$ of an $r$ dimensional space. In the general case, when designing a machine, one has to take into account the design variable constraints, the functional constraints, and the criteria constraints [1].There also exist particular performance criteria, such as productivity, materials consumption, and efficiency. It is desired that, with other things being equal, these criteria, denoted by $\Phi_{v}(\alpha), v=1, \ldots, k$, would have the extreme values. For simplicity, we assume that $\Phi_{v}(\alpha)$, are to be minimized.

In order to avoid situations in which the expert regards the values of some criteria as unacceptable, we introduce the criteria constraints

$$
\Phi_{v}(\alpha) \leq \Phi_{v}^{* *}, v=1, \ldots, k
$$

\footnotetext{
* Corresponding author: matusoff.1@yandex.ru
} 
where $\Phi_{v}^{* *}$ is the worst value of criterion $\Phi_{v}(\alpha)$ to which the expert may agree.

The criteria constraints differ from the functional constraints in that the former are determined when solving a problem and, as a rule, are repeatedly revised. Hence, reasonable values of $\Phi_{v}^{* *}$ cannot be chosen before solving the problem.

The design variable constraints, the functional constraints and the criteria constraints define the feasible solution set $D[1,2]$.

Let us formulate one of the basic problems of multicriteria optimization.

Definition 1. A point $\alpha^{0} \in D$, is called the Pareto optimal point if there exists no point $\alpha$ $\in D$ such that $\Phi_{v}(\alpha) \leq \Phi_{v}\left(\alpha^{0}\right)$ for all $v=1, \ldots, k$, and $\Phi_{v_{0}}(\alpha)<\Phi_{v_{0}}\left(\alpha^{0}\right)$ for at least one $v_{0} \in\{1, \ldots, k\}$.

A set $P \subset D$ is called the Pareto optimal set if it consists of Pareto optimal points. When solving the problem, one has to determine a design variable vector point $\alpha^{0} \in P$, which is most preferable among the vectors belonging to set $P$.

The Pareto optimal set plays an important role in vector optimization problems because it can be analyzed more easily than the feasible solution set and because the optimal vector always belongs to the Pareto optimal set, irrespective of the system of preferences used by the expert for comparing vectors belonging to the feasible solution set.

\section{Approximation of the feasible set}

\subsection{The estimation of the convergence rate}

The algorithm discussed in [2] allows simple and efficient identification and selection of feasible points from the design variable space. However, the following question arises: How can one use the algorithm to construct a feasible solution set $D$ with a given accuracy? The latter is constructed by singling out a subset of $D$ that approaches any value of each criterion in region $\Phi(D)$ with a predetermined accuracy. Let $\varepsilon_{v}$ be an admissible (in the expert's opinion) error in criterion $\Phi_{v}$. By $\varepsilon$ we denote the error set $\left\{\varepsilon_{v}\right\}, v=1, \ldots, k$. We will say that region $\Phi(D)$ is approximated by a finite set $\Phi\left(D_{\varepsilon}\right)$ with an accuracy up to the set $\varepsilon$, if for any vector $\alpha \in D$, there can be found a vector $\beta \in D_{\varepsilon}$ such that

$$
\left|\Phi_{v}(\alpha)-\Phi_{v}(\beta)\right| \leq \varepsilon_{v}, \quad v=1, \ldots, k .
$$

We assume that the functions we shall be operating with are continuous and satisfy the Lipschitz condition ( $L$ ) formulated as follows: For all vectors $\alpha$ and $\beta$ belonging to the domain of definition of the criterion $\Phi_{v}(\alpha)$, there exists a number $L_{v}$ such that

$$
\left|\Phi_{v}(\alpha)-\Phi_{v}(\beta)\right| \leq L_{v} \max _{j}\left|\alpha_{j}-\beta_{j}\right| .
$$

In other words, there exists $L_{V}^{\prime}$ such that

$$
\left|\Phi_{v}(\alpha)-\Phi_{v}(\beta)\right| \leq L_{v}^{\prime} \sum_{j=1}^{r}\left|\alpha_{j}-\beta_{j}\right| .
$$

We will say that a function $\Phi_{v}(\alpha)$ satisfies the special Lipschitz condition $(S L)$ if for all 
vectors $\alpha$ and $\beta$ there exist numbers $L_{v}^{j}, j=1, \ldots, r$ such that

$$
\left|\Phi_{v}(\alpha)-\Phi_{v}(\beta)\right| \leq \sum_{j=1}^{r} L_{v}^{j}\left|\alpha_{j}-\beta_{j}\right|
$$

where at least some of the $L_{v}^{j}$ are different.

Let $\left[L_{v}\right]$ (or $\left[\sum_{j=1}^{r} L_{v}^{j}\right]$ ) be a dyadic rational number exceeding $L_{v}$ (or $\sum_{j=1}^{r} L_{v}^{j}$ ) and sufficiently close to the latter, and let $\left[\varepsilon_{v}\right]$ be the maximum dyadic rational number that is less than or equal to $\varepsilon_{v}$ and whose numerator is the same as that of $\left[L_{v}\right]\left(\right.$ or $\left.\left[\sum_{j=1}^{r} L_{v}^{j}\right]\right)$. A dyadic number is a number of the form $p / 2^{m}$, where $p$ and $m$ are natural numbers.

Theorem 1. If criteria $\Phi_{v}(\alpha)$ are continuous and satisfy either the Lipschitz condition or the special Lipschitz condition, then to approximate $\Phi(D)$ to within an accuracy of $\varepsilon$ it is sufficient to have

$$
\max _{v} 2^{\tau}\left(\frac{\left[L_{v}\right]}{\left[\varepsilon_{v}\right]}\right)^{r} \text { or } \max _{v} 2^{\tau}\left(\frac{\left[\sum_{j=1}^{r} L_{v}^{j}\right]}{\left[\varepsilon_{v}\right]}\right)^{r}
$$

points of the $\mathrm{P}_{\tau}$ net [3].( For details on $\tau$ and the $\mathrm{P}_{\tau}$ net, see [1].)

The number of points needed to calculate the performance criteria in this estimate may be so large that the speed of computers may prove to be inadequate. This difficulty may be overcome by developing "fast" algorithms dealing not with an entire class of functions but instead taking into account the features of the functions of each concrete problem.

\subsection{The algorithm of approximation}

Let the Lipschitz constants $L_{v} v=1, \ldots, k$, be specified, and let $N_{1}$ be the subset of the points of $D$ that are either the Pareto optimal points or lie within the $\varepsilon$-neighborhood of a Pareto optimal point with respect to at least one criterion. In other words, $\Phi_{v}\left(\alpha^{0}\right) \leq \Phi_{v}(\alpha) \leq$ $\Phi_{v}\left(\alpha^{0}\right)+\varepsilon_{v}$, where $\alpha^{0} \in P$, and $P$ is the Pareto optimal set. Also, let $N_{2}=D \backslash N_{1}$ and $\bar{\varepsilon}_{v}>\varepsilon_{v}$, .

Definition 2. A feasible solution set $\Phi(D)$ is said to be normally approximated if any point of set $N_{1}$ is approximated to within an accuracy of $\varepsilon$, and any point of set $N_{2}$ to within an accuracy of $\bar{\varepsilon}$. In next theorem the algorithm of approximation is given.

Theorem 2. There exists a normal approximation $\Phi\left(D_{\varepsilon}\right)$ of a feasible solution set $\Phi(D)$. 


\section{Multicriteria modeling (identification)}

As a rule these applied identification problems have been treated as single-criterion problems. In the majority of conventional problems, the system is tacitly assumed to be in full agreement with its mathematical model. However, for complex engineering systems (e.g., machines) we generally cannot assert a sufficient correspondence between the model and the object. This does not permit us to use a single criterion to evaluate the adequacy. In multicriteria identification problems there is no necessity of artificially introducing a single criterion to the detriment of the physical essence of the problem.

Parametric identification is reduced to finding numerical values of the equation coefficients, based on the realization of the input and output processes. In doing so, frequency responses, transfer functions, and unit step functions are often used. A number of problems require preliminary experimental determination of the basic characteristics of a mechanical system (e.g., the frequencies, shapes, and decrements of natural oscillations). In identification problems we will deal with particular adequacy (proximity) criteria. By adequacy (proximity) criteria we mean the discrepancies between the experimental and computed data, the latter being determined on the basis of the mathematical model. For example, when identifying the parameters of the dynamical model of an automobile it is necessary to take into account such important indices (particular criteria) as vibration accelerations at all characteristic points of the driver's seat, driver's cab, frame, and engine; vertical dynamical reactions at contact areas between the wheels and the road; relative (with respect to the frame) displacements of the cab, wheels, engine, etc. [3]

In all basic units of the structure under study we experimentally measure the values of the characteristic quantities of interest (e.g., displacements, velocities, accelerations, etc.). At the same time we calculate the corresponding quantities by using the mathematical model. As a result, particular adequacy (proximity) criteria are formed as functions of the difference between the experimental and computed data. Thus we arrive at a multicriteria problem. The multicriteria consideration makes it possible to extend the application area of the identification theory substantially.

\subsection{Defining a feasible solution set and the adequate vectors}

We denote by $\Phi_{v}^{c}(\alpha), v=\overline{1, k}$ the indices (criteria) resulting from the analysis of the mathematical model that describes a physical system, where $\alpha=\left(\alpha_{1}, \ldots, \alpha_{r}\right)$ is the vector of the parameters of the model. Let $\Phi_{v}^{\exp }$ be the experimental value of the criterion measured directly on the prototype. The experiment is assumed to be sufficiently accurate and complete. Suppose there exists a mathematical model or a hierarchical set of models describing the system behavior. Let $\Phi=\left(\left\|\Phi_{1}^{c}-\Phi_{1}^{\exp }\right\|, \ldots,\left\|\Phi_{k}^{c}-\Phi_{k}^{\exp }\right\|\right)$, where $\|$. is a particular adequacy (closeness, proximity) criterion. This criterion, as has already been mentioned, is a function of the difference (error) $\Phi_{v}^{c}-\Phi_{v}^{\exp }$. Very often it is given by $\left(\Phi_{v}^{c}-\Phi_{v}^{\exp }\right)^{2}$ or $\left|\Phi_{v}^{c}-\Phi_{v}^{\exp }\right|$. If the experimental values $\Phi_{v}^{\exp }, v=\overline{1, k}$ are measured with considerable error, then the quantity $\Phi_{V}^{\exp }$ can be treated as a random variable. If this random variable is normally distributed, the corresponding adequacy criterion is expressed by $M\left\{\Phi_{v}^{c}-\Phi_{v}^{\exp \|\}}\right.$, where $M\{\|\cdot\|\}$ denotes the mathematical expectation of the 
random variable $\|$.$\| . For other distribution functions, more complicated methods of$ estimation are used, for example, the maximum likelihood method.

We formulate the following problem by comparing the experimental and calculation data, determining to what extent the model corresponds to the physical system, and finding the variables of the model. In other words, it is necessary to find the vectors $\alpha^{i}$ satisfying the design variable constraints, the functional constraints, and the next criteria constraints

$$
\left\|\Phi_{v}^{c}\left(\alpha^{i}\right)-\Phi_{v}^{\exp }\right\| \leq \Phi_{v}^{* *}
$$

These constraints define the feasible solution set $D_{\alpha}$. Here, $\Phi_{v}^{* *}$ are criteria constraints that are determined in the dialogue between the researcher and a computer. To a considerable extent, these constraints depend on the accuracy of the experiment and the physical sense of the criteria. The formulation and solution of the identification problem are based on the parameter space investigation method. We specify the values $\Phi_{v}^{* *}$ and find vectors meeting the design variable constraints, the functional constraints, and the criteria constraints. The vectors $\alpha_{i d}^{i}$ belonging to the feasible solution set $D_{\alpha}$ will be called adequate vectors [4-6].

The restoration of the parameters of a specific model is the main purpose and essence of multicriteria parametric identification. Having performed this procedure for all structures (mathematical models), we thus carry out multicriteria structural identification.

The vectors $\alpha_{i d}^{i}$ that belong to the set of adequate vectors and have been chosen by using a special decision making rule will be called identified vectors.

The role of the decision making rule is often played by nonformal analysis of the set of adequate vectors. If this analysis separates several equally acceptable vectors $\alpha_{i d}^{i}$, the solution of the identification problem is nonunique.

The identified vectors $\alpha_{i d}^{i}$ form the identification domain $D_{i d}=\bigcup_{i} \alpha_{i d}^{i}$. Sometimes, by carrying out additional physical experiments, revising constraints $\Phi_{v}^{* *}$, etc., one can reduce the domain $D_{i d}$ and even achieve the result that this domain contains only one vector. Unfortunately, this is far from usual. Nonunique restoration of variables is a recompense for the discrepancy between the physical object and its mathematical model, incompleteness of physical experiments, etc.

If a mathematical model is sufficiently good (i.e., it correctly describes the behavior of the physical system), then multicriteria parametric identification leads to a nonempty set $D_{\alpha}$. The most important factors that can lead to an empty $D_{\alpha}$ are imperfection of the mathematical model and lack of information about the domain in which the desired solutions should be searched for.

The search for the set $D_{\alpha}$ is very important, even in the case where the results are not promising. It enables the researcher to judge the mathematical model objectively (not only intuitively), to analyze its advantages and drawbacks on the basis of all proximity criteria. 


\subsection{The search for identified solutions with the prescribed accuracy}

Let $\varepsilon_{v}(v=\overline{1, k)}$ characterize the desired accuracy of the correspondence between the physical system and its mathematical model with respect to the criterion $\Phi_{v}^{c}$ (i.e., the inequality $\left\|\Phi_{v}^{c}-\Phi_{v}^{\exp }\right\|<\varepsilon_{v}$ must hold). Then the values of all criteria restoring the experimental characteristics with a prescribed accuracy can be found through the approximation of the adequacy criteria range.

In multicriteria identification, we are interested not only in values of adequacy criteria, but also in values of variables. For example, let $\alpha$ and $\beta$ be vectors giving "good" values to adequacy criteria, i.e., $\Phi(\alpha) \approx \Phi(\beta)$, while at the same time the vectors $\alpha$ and $\beta$ are significantly different. In this case, if there is no additional information available for making the choice between the vectors $\alpha$ and $\beta$, we can regard $\Phi(\alpha), \Phi(\beta)$ as being equally adequate to the physical experiment. However, the researcher must keep in mind all vectors corresponding to good values of adequacy criteria. This is explained by the following considerations. In practice, it is usually impossible to formalize all requirements imposed on a physical or engineering system. If we take into account only one of two vectors corresponding to approximately the same values of adequacy criteria, we may possibly lose the better vector with respect to nonformalized criteria. Suppose we have succeeded in meeting all the demands of the system. In this case, we should consider all the aforementioned vectors when working with the mathematical model after completing the identification. Suppose we are to optimize the parameters of the model with respect to some criteria. If we have eliminated one of two equally adequate vectors, the dropped vector can turn out to be the preferred one with regard to the performance criteria. Taking into account these considerations, we can modify the definition of the solution of the multicriteria identification problem.

We denote by $V_{\varepsilon}(\Phi(P))$ an $\varepsilon$-neighborhood of the Pareto optimal set $\Phi(P)$ in the space of adequacy criteria [7]. It is reasonable to define the solution of the multicriteria identification problem as a set $W_{\varepsilon}$ of all variable vectors $\alpha$ belonging to the feasible solution set $D_{\alpha}$ and satisfying the inclusion $\Phi(\alpha) \in V_{\varepsilon}(\Phi(P))$.

As a result of nonformal analysis of the set $W_{\varepsilon}$, the researcher can choose the most preferred vectors.

Let us show how one can solve the problem by using the parameter space investigation method.

The solution algorithm is based not only on the approximation of the criteria space, but also on the approximation of the variable space. Let $\Phi_{k+j}(\alpha)=\alpha_{j}$, and $\delta_{k+j}$ be the admissible error for the variable $\alpha_{j}$, where $k$ is the number of adequacy criteria. By using the algorithm of Theorem 2, let us construct the approximation of the set $D_{\alpha}$ to the accuracy $\delta=\left\{\delta_{k+j}\right\}, j=\overline{1, r}$, and the approximation of its image $\Phi\left(D_{\alpha}\right)$, to the accuracy $\varepsilon=\left\{\varepsilon_{v}\right\}, v=\overline{1, k}$. The fact that we have declared the variables $\alpha_{j}$ as criteria $\Phi_{k+j}$, enables us to approximate $\Phi\left(D_{\alpha}\right)$ and $D_{\alpha}$ simultaneously. In this case, the set $V_{\varepsilon}(\Phi(P))$ can be approximated to the accuracy $\varepsilon$, and any vector of $D_{\alpha}$ can be determined to the accuracy $\delta$ Using the approximations of $D_{\alpha}$, and $\Phi\left(D_{\alpha}\right)$ we can find the set $W_{\varepsilon}$, and thus obtain the solution of the multicriteria identification problem. 
Let us call the set $W_{\varepsilon}$ the set of $\varepsilon$-adequate vectors. The vectors $\alpha_{i d}$ that belong to the set of $\varepsilon$-adequate vectors and are determined with the help of a decision-making rule will be called identified vectors. The set $D_{i d}$ of all identified vectors is called the identification set.

\section{Conclusions}

The formulation and solution of the multicriteria optimization and identification problems combined with nonformal analysis of the results make it possible to:

1.Determine the feasible set, the sets of adequate and identified solutions with prescribed accuracy and thus assess the agreement between the technical system and its mathematical model.

2.Determine the advantages of one or another model.

3.Correctly specify, on establishing the adequacy of the mathematical model, the boundaries of the design variable range and justify the list of performance criteria.

\section{References}

1. I.M. Sobol', R.B. Statnikov, The Best Solutions: Where They May Be Found, Moscow, (1982.)

2. I. Sobol', R. Statnikov, Selecting Optimal Parameters in Multicriteria Problems, Moscow, (2006).

3. J.B. Matusov, R.B. Statnikov, Approximation and vector optimization of large systems, Doklady AN SSSR, V. 296(3),pp 532-536, (1987)

4. R.B. Statnikov, J.B. Matusov, Multicriteria Machine Design, Moscow, (1989).

5. R.B. Statnikov, J.B. Matusov, Multicriteria Optimization and Engineering, New York,(!995).

6. R.B. Statnikov, J.B. Matusov, Use of P-nets for the approximation of the EdgeworthPareto set in multicriteria optimization, JOTA, V. 91, (3), pp. 543-560,(1996).

7. R. Statnikov, J. Matusov, Multicriteria Analysis in Engineering, Dordrecht,(2002). 